\title{
DENSE IMBEDDING OF TEST FUNCTIONS IN CERTAIN FUNCTION SPACES
}

\author{
MICHAEL RENARDY
}

\begin{abstract}
In a recent paper [1], J. U. Kim studies the Cauchy problem for the motion of a Bingham fluid in $R^{2}$. He points out that the extension of his results to three dimensions depends on proving the denseness of $C^{\infty}$-functions with compact support in certain spaces. In this note, such a result is proved.
\end{abstract}

Following Kim's notation [1], we define the following spaces:

$$
\begin{aligned}
& \tilde{F}_{p}\left(R^{n}\right)=\left\{u \in W^{1,2}\left(R^{n}\right) \mid \nabla u \in\left(L^{p}\left(R^{n}\right)\right)^{n}\right\}, \\
& F_{p}\left(R^{n}\right)=\left\{u \in\left(W^{1,2}\left(R^{n}\right)\right)^{n} \mid \nabla u \in\left(L^{p}\left(R^{n}\right)\right)^{n \times n}, \operatorname{div} u=0\right\}, \\
& G_{p}\left(R^{n}\right)=\left\{u \in\left(W^{1,2}\left(R^{n}\right)\right)^{n} \mid \varepsilon(u)=\nabla u+(\nabla u)^{T} \in\left(L^{p}\left(R^{n}\right)\right)^{n \times n}, \operatorname{div} u=0\right\}, \\
& S\left(R^{n}\right)=\left\{u \in\left(C_{0}^{\infty}\left(R^{n}\right)\right)^{n} \mid \operatorname{div} u=0\right\} .
\end{aligned}
$$

According to Kim's Lemma 1.7 [1], $F_{p}=G_{p}$ for $1<p<\infty$. The results, which will be presented in this paper, are the following.

THEOREM 1. Let $n$ be arbitrary and $1 \leqslant p<\infty$. Then $C_{0}^{\infty}\left(R^{n}\right)$ is dense in $\tilde{F}_{p}\left(R^{n}\right)$.

TheOREM 2. Let $n=2$ or $n=3$ and $1 \leqslant p<\infty$. Then $S\left(R^{n}\right)$ is dense in $F_{p}\left(R^{n}\right)$ and $G_{p}\left(R^{n}\right)$.

We remark that the case $p=2$ of Theorem 2 is well known, even in the context of general domains (see, for example, Heywood [2]). The proofs of both theorems will make use of the following lemma.

Lemma. For $x \in R^{n}$, let

$$
\phi_{N}(x)= \begin{cases}\left(N^{n} \Omega_{n}\right)^{-1} & \text { if }|x| \leqslant N, \\ 0 & \text { if }|x|>N,\end{cases}
$$

where $\Omega_{n}$ denotes the volume of the unit ball in $R^{n}$. Let $1 \leqslant r<\infty$ and $v \in L^{r}\left(R^{n}\right)$; if $r=1$, assume in addition that $\int_{R^{n}} v=0$. Then $\phi_{N} * v \rightarrow 0$ in $L^{r}\left(R^{n}\right)$ as $N \rightarrow \infty$.

Received by the editors November 22, 1985.

1980 Mathematics Subject Classification. Primary 46E35.

Key words and phrases. Sobolev spaces, approximation by test functions.

${ }^{1}$ The author was sponsored by the United States Army under Contract No. DAAG29-80-C-0041 and by the National Science Foundation under Grants No. MCS-8215064 and DMS-8451761. 
Proof of THE Lemma. Since $\left\|\phi_{N}\right\|_{L^{1}}=1$, we have $\left\|\phi_{N} * v\right\|_{L^{r}} \leqslant\|v\|_{L^{r}}$, and hence it suffices to show that $\phi_{N} * v \rightarrow 0$ for $v$ in a dense subset of $L^{r}$. If $r>1$, take $v \in L^{1} \cap L^{r}$. Then $\left\|\phi_{N} * v\right\|_{L^{r}} \leqslant\left\|\phi_{N}\right\|_{L^{r}}\|v\|_{L^{1}}$, which tends to zero as $N \rightarrow \infty$. For $r=1$, let $v$ have compact support, contained in, say, $\{|x| \leqslant R\}$, and assume $\int_{R^{n}} v=0$. Then

$$
\begin{aligned}
\left\|\phi_{N} * v\right\|_{L^{1}} & =\int_{R^{n}}\left|\int_{R^{n}} \phi_{N}(x-y) v(y) d y\right| d x \\
& =\int_{N-R \leqslant|x| \leqslant N+R}\left|\int_{|y| \leqslant R} \phi_{N}(x-y) v(y) d y\right| d x \\
& \leqslant \int_{N-R \leqslant|x| \leqslant N+R} \int_{|y| \leqslant R}\left|\phi_{N}(x-y)\right||v(y)| d y d x \\
& \leqslant \int_{N-2 R \leqslant|z| \leqslant N}\left|\phi_{N}(z)\right| d z \cdot \int_{|y| \leqslant R}|v(y)| d y .
\end{aligned}
$$

This tends to zero as $N \rightarrow \infty$.

Proof of Theorem 1. Clearly it suffices to show that functions of compact support are dense, $C^{\infty}$-regularity can easily be achieved by using a mollifier. If we know that $u \in L^{p}\left(R^{n}\right)$ or even that $u \in L^{p+\varepsilon}\left(R^{n}\right)$ for small enough $\varepsilon>0$, then we can use the standard cut-off procedure to approximate $u$ by functions of compact support, i.e., if we set $u_{m}(x)=u(x) \psi_{m}(x)$, where, for example,

$$
\psi_{m}(x)= \begin{cases}1 & \text { if }|x| \leqslant m, \\ 2-|x| / m & \text { if } m \leqslant|x| \leqslant 2 m, \\ 0 & \text { if }|x| \geqslant 2 m,\end{cases}
$$

then it is easy to show that $u_{m} \rightarrow u$ in $\tilde{F}_{p}$. Therefore, it suffices to show that $\tilde{F}_{p} \cap L^{p+\varepsilon}(\varepsilon \geqslant 0$ small $)$ is dense in $\tilde{F}_{p}$. If $p \geqslant 2$, then the Sobolev imbedding theorem can be used to show that $\tilde{F}_{p} \subset L^{p}$, and there is nothing left to prove.

For $p<2$, let $\phi_{N}$ be as in the lemma above. For $u \in \tilde{F}_{p}$, let $u_{N}=u-\phi_{N} * u$. We have $\nabla u_{N}=\nabla u-\phi_{N} * \nabla u$, and, if $p=1$, then $\int_{R^{n}} \nabla u=0$, since $u \in L^{2}$. Therefore, the lemma implies that $u_{N} \rightarrow u$ as $N \rightarrow \infty$ in the norm of $\tilde{F}_{p}$. It is therefore enough to show that $u_{N}$ lies in $L^{p+\varepsilon}$ for small $\varepsilon>0$. Let $g$ denote the fundamental solution for Laplace's equation,

$$
g(x)= \begin{cases}-|x|^{2-n} / \omega_{n}(n-2) & \text { if } n \geqslant 3 \\ \ln |x| / 2 \pi & \text { if } n=2\end{cases}
$$

where $\omega_{n}$ denotes the surface measure of the unit sphere in $R^{n}$. In any dimension, $g$ and its first derivatives are in $L_{\text {loc }}^{1+\delta}$ for sufficiently small $\delta \geqslant 0$. We want to consider the behavior of $g-\phi_{N} * g$ at infinity. We have

$$
g(x)-\phi_{N} * g(x)=g(x)-\int_{|y-x|<N} \frac{g(y)}{N^{n} \Omega_{n}} d y .
$$


By expanding the integrand in a Taylor series about $x$, we find that this can be bounded by a constant times

$$
N^{2} \max _{|y-x| \leqslant N} \max _{i, j}\left|\frac{\partial^{2} g(y)}{\partial x_{i} \partial x_{j}}\right| .
$$

Since second derivatives of $g$ decay like $|x|^{-n}$ at infinity, it follows that $g-\phi_{N} * g$ is in $L^{1+\delta}$ at infinity for any positive $\delta$, and so are derivatives of $g$ by the same argument. Hence we conclude that, for small enough $\delta>0, g-\phi_{N}^{*} g$ lies in $L^{1+\delta}$. It follows that $\omega_{N}=g * \nabla u_{N}=\left(g-\phi_{N} * g\right) * \nabla u$ lies in $L^{p+\varepsilon}$ for small positive $\varepsilon$, and so do its first derivatives. Since $\operatorname{div} \omega_{N}=u_{N}$, this completes the proof.

Proof of Theorem 2. For $p>1$, the arguments used by Kim [1] show that Theorem 2 follows from Theorem 1 . We may hence concentrate on the case $p=1$. For $u \in F_{1}$ or $G_{1}$, let $u_{N}=u-\phi_{N} * u$ with $\phi_{N}$ as before. As in the proof of Theorem 1, it can be shown that $u_{N} \rightarrow u$ in $F_{1}$ or $G_{1}$, respectively. Moreover, let $a_{N}=g * \operatorname{curl} u_{N}=\left(g-\phi_{N} * g\right) * \operatorname{curl} u$. The convolution $g * \operatorname{curl} u_{N}$ makes sense because $G_{1}$ and $F_{1}$ are contained in $F_{p}$ for $1<p \leqslant 2$, hence the same argument as in the proof of Theorem 1 shows that curl $u_{N}$ as well as $u_{N}$ are in $L^{p}$ for $p \in(1,2]$. Moreover, $g$ is integrable at the origin, and its derivative has some power that is integrable at infinity. We can thus decompose $g$ in the form $g=g_{1}+g_{2}$, where $g_{1} \in L^{1}$ and $\nabla g_{2} \in L^{q}$ for some $q<\infty$. Clearly $g_{1} *$ curl $u_{N}$ is defined, and $g_{2} * \operatorname{curl} u_{N}$ can be defined by transferring the derivative onto $g_{2}$. We have $\Delta a_{N}=$ $\operatorname{curl} u_{N}$ and $\operatorname{curl} a_{N}=g * \operatorname{curl} \operatorname{curl} u_{N}=g *\left(-\Delta u_{N}\right)=-u_{N}$. Since $G_{1}$ and $F_{1}$ are contained in $F_{p}$ for every $p \in(1,2]$, curl $u$ lies in $L^{1+\varepsilon}$ for $0<\varepsilon \leqslant 1$, and we can conclude as in the proof of Theorem 1 that

$$
a_{N}=\left(g-\phi_{N} * g\right) * \operatorname{curl} u \in L^{1+\varepsilon}
$$

Since $\Delta a_{N}$ is also in $L^{1+\varepsilon}$, it follows that $a_{N} \in W^{2,1+\varepsilon}$.

It thus remains to show that every $u \in G_{1}$ or $F_{1}$ which has the form $u=\operatorname{curl} a$ with $a \in W^{2,1+\varepsilon}$ can be approximated by functions with compact support. This can easily be achieved by multiplying $a$ with a suitable cut-off function.

\section{REFERENCES}

1. J. U. Kim, On the Cauchy problem associated with the motion of a Bingham fluid in the plane, Trans. Amer. Math. Soc. (to appear).

2. J. G. Heywood, On uniqueness questions in the theory of viscous flow, Acta Math. 136 (1976), 61-102.

Department of Mathematics and Mathematics Research Center, University of Wisconsin, MADISON, WISCONSIN 53706

Current address: Department of Mathematics, Virginia Polytechnic Institute and State University, Blacksburg, Virginia 24061 